\title{
Cooperación judicial e integración subnacional chileno-argentina: principales logros y avances ${ }^{1}$
}

Judicial cooperation and sub-national integration ChileArgentina: main achievements and developments

\section{Alejandro Sfeir Tonsic}

alesfeir@hotmail.com

Abogado de la Universidad de Chile. Máster en Política Exterior de la Universidad de Santiago de Chile.

Resumen: La cooperación judicial desarrollada entre diversos órganos de las provincias argentinas y regiones chilenas, al amparo del Tratado de Maipú de Integración y Cooperación de 2009 y de los Comités de Integración binacionales, ha experimentado sustantivos avances en los últimos años, los cuales se ven reflejados en las diversas actividades que se describen en el artículo, las cuales han sido coordinadas y lideradas por las cancillerías de ambos países.

Por su parte, la cooperación judicial binacional se entrelaza con el concepto de integración subnacional tanto al permitir un amplio encuentro de altos funcionarios y profesionales interesados en avanzar en estas materias, como al hacer posible el estrechamiento de los vínculos ya existentes a ambos lados de la Cordillera de los Andes.

Como fruto de las conversaciones mantenidas en Mendoza en el Comité "Paso Cristo Redentor" de 1 y 2 de septiembre de 2011 y de la organización del Primer Seminario Internacional de Cooperación Judicial Chile-Argentina, desarrollado el 29 y 30 de marzo de 2012, se lograron implementar nuevas Comisiones de Cooperación Judicial en los demás Comités de Integración, así como organizar nuevos seminarios sobre dicha temática, con el objeto de avanzar en el campo de la integracion y la cooperación judicial. El objetivo del presente artículo es, precisamente, dar cuenta de los principales logros y avances en esa materia.

Palabras clave: cooperación judicial, integración subnacional, comités de integración, Tratado de Maipú.

Abstract: The judicial cooperation developed between several organs of the Argentine provinces and regions of Chile, under the Treaty of Maipu on Integration and Cooperation (2009) and the Committees of bi-national integration, has experienced substantial progress in recent years, which are reflected in different activities described in this article, which have been coordinated and led by the Foreign Ministries of the two countries. Meanwhile, the bilateral judicial cooperation is intertwined with the concept of sub-national integration by allowing both a broad meeting of senior officials and professionals interested in advancing in these areas, as to make

Artículo recibido el 13.11.2013 y aceptado el 15.10.2014. 
possible the narrowing of the existing links on both sides of the Andes. As a result of discussions in Mendoza in the "Cristo Redentor" Committee on 1 and 2 September 2011, and the organization of the First International Seminar on Judicial Cooperation Chile-Argentina on 29 and 30 March 2012 were able to implement new Commissions on Judicial cooperation in other Integration Committees and organize new seminars on such issue, in order to advance the field of judicial cooperation and integration. This article aims to report on the main achievements and developments in this area.

Keywords: judicial cooperation, sub-national integration, integration committees, Maipu Treaty.

\section{Aspectos generales ${ }^{2}$}

El desafío de desentrañar los verdaderos alcances actuales y futuros de la cooperación judicial chileno-argentina resulta, además de muy necesario, del mayor interés en los tiempos que corren.

A su vez, si esta circunstancia histórica la insertamos dentro del complejo y exitoso proceso de integración subnacional por el que atraviesan estos dos Estados sudamericanos, sólo entonces la temática abordada alcanza ribetes particularmente interesantes, tanto por sus implicancias como por sus positivas consecuencias para estas dos naciones hermanas.

Tal aseveración se apoya en la convicción de que todo proceso de integración, en cualquiera de sus variadas expresiones, sólo puede generar los frutos y efectos esperados si está amparado y regulado por un ordenamiento jurídico afín, parte del cual se refleja en un eficiente y perfectible proceso de cooperación judicial.

En tal sentido, cualquier duda existente sobre la real utilidad o potenciales efectos benéficos de tal proceso sucumbiría frente a la constatación de los avances experimentados por el mismo, máxime si aquellos resultan evidentes a la observación de cualquier interesado.

Por ello, y para abordar conceptualmente esta temática, señalemos preliminarmente que por cooperación judicial y/o jurídica nos referimos al mecanismo por medio del cual dos o más Estados se prestan colaboración mutua, con el propósito de abordar y resolver satisfactoriamente las diferentes necesidades surgidas de la sustanciación de procesos judiciales que tienen origen, interés o consecuencias en más de un Estado.

2 Las opiniones de este artículo sólo representan la personal óptica académica del autor como expositor en el Primer Seminario Internacional de Cooperación Judicial Chile-Argentina, de 29 y 30 de marzo de 2012, desarrollado en Mendoza, en su calidad de egresado del Doctorado en Derecho Internacional de la Facultad de Derecho, de la Universidad de Buenos Aires. 
Resulta conocido pues que la justicia debe ser rápida y oportuna, pues la justicia que tarda no cumple su rol de pacificación social. Por su parte, también sabemos que el cumplimiento de las resoluciones judiciales en el extranjero resulta notablemente más complejo y demorado que en el propio territorio nacional. Consecuentemente, es aquí donde el concepto de cooperación internacional entra a jugar un papel clave para que la justicia cumpla -en tiempo y forma- su rol natural.

Por ello, resulta útil analizar someramente algunas recientes iniciativas adoptadas en la materia -como la realización de seminarios, mesas de trabajo y otros tipos de encuentros judiciales ${ }^{3}$-, las cuales han permitido dinamizar tal estrecha cooperación, al hacerla aún más efectiva y geográficamente más localizada, en el marco del pleno respeto de la normativa internacional vigente.

En este punto valga señalar que aquí no nos estamos refiriendo a la cooperación jurídica existente en el ámbito bilateral general, llevada eficientemente a cabo por las autoridades centrales de las cancillerías, sino que aludimos a la cooperación judicial a nivel subnacional.

\section{El Tratado de Maipú}

La integración subnacional chileno-argentina es definida como aquella donde trabajan los gobiernos, órganos regionales y entidades de la sociedad civil de las provincias argentinas y regiones chilenas, situadas a uno y otro lado de la Cordillera de Los Andes ${ }^{4}$. Ciertamente que, según el Tratado de Integración y Cooperación de Maipú de 30 de octubre de 2009, tal integración es, también, liderada y coordinada por las respectivas cancillerías.

En este contexto se puede constatar que Chile y Argentina, siendo países conscientes de los problemas que genera la globalización y la mayor circulación de personas, bienes, servicios y capitales, han avanzado seńeramente, utilizando variadas formas de cooperación, usando para ello tanto los procedimientos tradicionales vigentes, como las nuevas instancias de reflexión creadas por el propio Tratado de Maipú, el cual innova para utilizar aquellos que, dentro de la juridicidad existente, satisfacen plenamente las nuevas necesidades imperantes. Este es el caso de los denominados Comités de Integración.

3 Estas iniciativas fueron propuestas por el Ex Embajador de Chile en Argentina, Don Adolfo Zaldívar Larraín.

4 Maira, Luis. La Politica Internacional Subnacional en América Latina, Editorial Libros del Zorzal, Buenos Aires, 2010. 


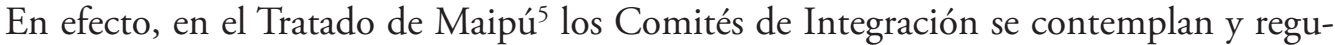
lan a partir del artículo $3^{\circ}$, considerándoseles como parte integrante de los diversos mecanismos institucionales de la integración. A su vez, el artículo $15^{\circ}$ los define como "foros de encuentro y colaboración entre sectores público y privado de las provincias argentinas y regiones chilenas, para promover la integración en el ámbito subnacional, con el apoyo de organismos nacionales, provinciales, regionales y municipales. Serán coordinados por los correspondientes Ministerios de Relaciones Exteriores a través de los organismos pertinentes, con la participación de los Cónsules respectivos, de acuerdo con las funciones que les asigna el Reglamento de los Comités de Integración, y de los responsables específicos en cada Embajada”.

Por su parte, el artículo $18^{\circ}$, señala que "los comités de integración podrán impulsar, entre otras, las siguientes actividades de cooperación destinadas al área geográfica de su competencia: a) favorecer el diálogo entre representantes de las entidades locales de cada una de las provincias argentinas y regiones chilenas, con el propósito de mejorar la calidad de vida de sus habitantes. En este plano, los Comités de Integración constituyen mecanismos que apoyan y refuerzan las iniciativas vinculadas con el respeto y protección de la identidad cultural, derechos humanos, migraciones, inserción laboral, seguridad, justicia y educación"; y “c) En materia de Justicia y Seguridad, recomendar medidas que faciliten la colaboración de las fuerzas de seguridad pública en la prevención y lucha contra el delito, coordinadas con las autoridades nacionales de las Partes".

Finalmente, el artículo $30^{\circ}$, letra c), prescribe que la "cooperación binacional estará destinada a (...) promover y efectuar el seguimiento de las instancias de cooperación descentralizada existentes entre ambas partes, de conformidad con la organización política interna de cada una de ellas".

Es aquí, entonces, donde la cooperación judicial se entrelaza con el concepto de integración subnacional chileno-argentina, materia de este trabajo.

\section{Antecedentes inmediatos de la cooperación judicial subnacional}

Si revisamos históricamente los Comités de Integración (que en sus inicios se denominaban Comités de Frontera), evidenciamos que el área de justicia presentaba un menor tratamiento y evolución en relación a otras áreas de la integración subnacional,

$5 \quad$ En vigencia desde el 21 de enero de 2010. 
toda vez que luego de tres décadas de comités de integración, aún no se habían creado Comisiones de Cooperación Judicial (en adelante las «CCJ») generándose, de paso, un importante rezago en el tratamiento de los aspectos jurisdiccionales con respecto a otros, como los de carácter político, administrativo y/o fronterizo.

Es así como a partir de las visitas recíprocas, en Buenos Aires y Santiago, de los Presidentes de las Cortes Supremas Nacionales de ambos países, verificadas en $2011^{7}$, y de la creación de las primeras CCJ en los Comités de Integración ${ }^{8}$ durante ese mismo ańo, se han sucedido una serie de otros acontecimientos institucionales que han potenciado vivamente la aludida cooperación, toda vez que a tales actividades se han ido sumando numerosos funcionarios y voluntades de entes interesados en profundizar dicho proceso?.

\section{Primer seminario de cooperación judicial bilateral}

En ese escalamiento de aproximaciones y como fruto de las conversaciones mantenidas en el Comité "Paso Cristo Redentor", de 1 y 2 de septiembre de 2011, se ideó y organizó el Primer Seminario Internacional de Cooperación Judicial Chile-Argentina, que tuvo lugar en la ciudad de Mendoza, el 29 y 30 de marzo de 2012.

Es precisamente con aquella actividad que la cooperación judicial subnacional alcanza un punto álgido, ya que a propósito de tal seminario se concretó un amplísimo encuentro de jueces y altos magistrados de ambos países -sin precedentes conocidos-, con el objeto de avanzar en el campo de la integración y cooperación judicial.

Cabe hacer presente que el referido seminario fue co-organizado entre la Embajada de Chile en Argentina y la Corte Suprema de Mendoza, sin dejar de considerar a la Corte Suprema chilena, que contribuyó decisivamente al éxito de aquel encuentro al enviar una decena de Presidentes de Cortes de Apelaciones, jueces letrados y funcionarios administrativos, además de un Ministro de su propia Corte $^{10}$.

Con la sola excepción de dos gérmenes de comisiones muy específicas y sobre materias restringidas.

No existen registros históricos en ninguna de ambas Cortes, de haberse realizado con anterioridad visitas recíprocas consecutivas de sus Presidentes, por lo que se trataría -en este caso- de un hecho particularísimo. Los Presidentes Sres. Ricardo Lorenzetti y Milton Juica se visitaron mutuamente, tanto en Argentina como en Chile.

8 Ambas iniciativas fueron adoptadas por el Ex Embajador de Chile, don Adolfo Zaldívar Larraín, en 2011.

9 A la fecha, se estima en más de doscientas personas las que han participado de tales CCJ y seminarios.

10 Participó allí el Ex Presidente de la Corte Suprema, Ministro Milton Juica Arancibia. 
Para tener una idea más amplia sobre la importancia de dicha actividad, y de su significativa convocatoria, valga señalar que además de contarse con la presencia del Gobernador de la Provincia y de los embajadores de ambos países ${ }^{11}$, participaron muy altas autoridades, tales como los Presidentes de las Cortes Supremas de Mendoza, Salta, Córdoba, Tucumán y Jujuy, así como el ex Presidente y Ministro de la Corte Suprema chilena, y Ministros de las Cortes de Apelaciones de Santiago, Valparaíso, Temuco, Talca, Copiapó, Concepción y Punta Arenas.

En tal sentido, el Seminario en comento ha sido uno de los frutos de los diversos Comités de Integración, al interior de los cuales se habían creado las respectivas CCJ. Cabe destacar que todos los altos magistrados chilenos que habían participado en cinco CCJ de Comités de Integración anteriores (realizados en las ciudades de Catamarca, Mendoza, San Martín de los Andes, Malargüe y Punta Arenas) fueron comisionados, nuevamente, por la Corte Suprema chilena para participar en esta iniciativa ampliada.

Como panelistas participaron profesores universitarios de Mendoza y Buenos Aires, dos altos funcionarios de la Suprema Corte de Justicia de Mendoza, una Fiscal española experta en Cooperación Judicial Internacional, tres profesores chilenos pertenecientes a la Universidad de Chile y Universidad de Talca, y el autor de esta nota.

Los objetivos perseguidos por el Seminario fueron, entre otros, la realización de un análisis de la situación actual en materia de cooperación judicial, con el objeto de identificar nuevas áreas en las cuales se pudiese profundizar el proceso; además de favorecer el encuentro y conocimiento entre jueces de ambos países, con el fin de avanzar en el campo de la integración y cooperación judicial, a partir del cual podrían nacer ideas útiles para el trabajo de Cortes Supremas, Cancillerías, Ministerios de Justicia, y Ministerios Públicos de ambos Estados.

Fue así como luego de verificada esa actividad, la cooperación judicial entre Chile y Argentina, desde la óptica subnacional del Tratado de Maipú, ha experimentado un notable avance en relación con su estado anterior.

\section{Seminarios, comisiones (CCJ) y mesas de trabajo}

Loa avances reseñados pueden observarse en la realización de nuevos seminarios ${ }^{12}$, comisiones de cooperación judicial y mesas de trabajo, a los cuales han concurrido representantes de los poderes judiciales y ejecutivos.

11 Estuvieron presentes el Gobernador mendocino, Sr. Francisco Pérez, y los Embajadores Sres. Ginés González García y Adolfo Zaldívar Larraín.

12 Son los casos del Seminario de Justicia Constitucional en Argentina y Chile (de abril de 2012) y de Justicia Medioambiental (de agosto de 2013) organizados por la Corte Suprema de Salta, en esa misma ciudad, en conjunto con la Embajada de Chile en Argentina. 
En el ámbito de los Comités de Integración, las CCJ han sesionado en quince oportunidades, según se indica a continuación:

En 2011 sesionaron en los Comités de: "Paso Cristo Redentor", en Mendoza (1 y 2 de septiembre); "Los Lagos", en San Martín de Los Andes (28 y 29 de septiembre); "Atacalar", en Catamarca (8 y 9 de noviembre); "Paso Pehuenche", en Malargüe (29 y 30 de noviembre); e "Integración Austral", en Punta Arenas (17 y 18 de noviembre).

En 2012 lo hicieron en el Comité de Integración "NOA - Norte Grande", en Salta (10 y 11 de mayo); "Integración Austral", en Comodoro Rivadavia ( 2 y 3 de agosto); "Paso Cristo Redentor", en San Esteban de Los Andes (30 y 31 de agosto); "Atacalar", en Copiapó (5 y 6 de septiembre) y "Los Lagos", en Puerto Varas (26 y 27 de septiembre).

Finalmente, en 2013 las CCJ sesionaron dentro del Comité "Paso de Agua Negra", en San Juan (24 y 25 de abril); "Paso Cristo Redentor", en Mendoza (8 y 9 de mayo); "Integración Austral", en Coyhaique ( 15 y 16 de mayo); "Atacalar", en Río Hondo de Santiago del Estero (26 y 27 de agosto) y “Los Lagos”, en Bahía Blanca (5 y 6 de septiembre).

A las numerosas reuniones de las CCJ cabe agregar la celebración de dos mesas de trabajo, en las ciudades de Santiago de Chile (en mayo de 2012) y en Mendoza (en mayo de 2013).

En estas mesas se analizaron las diversas iniciativas originadas del diálogo, del intercambio de experiencias y del reconocimiento de las necesidades diagnosticadas en las diversas CCJ.

Numerosa y variada ha sido la temática abordada por las CCJ, donde junto con tratar ciertas materias de fondo, se ha hecho constar la necesidad de ampliar la convocatoria a las mismas, para que puedan enterarse y participar los diversos entes interesados en la materia, y, adicionalmente, dar mayor difusión a la misma. Asimismo, se ha conversado el tema relativo a la agilización de las notificaciones y las comunicaciones judiciales, lo cual resulta una necesidad permanente de la cooperación bilateral.

Particular relevancia ha tenido el análisis de ciertas instancias informativas sobre cooperación judicial a nivel iberoamericano (disponibles en la web), útiles a la relación judicial bilateral, siendo éstas: la Red Iberoamericana de Cooperación Jurídica Internacional (IberRed); el sistema de comunicación segura de IberRed (el “Iber@”); la Red Iberoamericana de Información y Documentación Judicial (IBERIUS); la Red Iberoamericana de Escuelas Judiciales (RIAEJ); el Centro Iberoamericano de Capacitación Judicial Virtual (CICJV) y el Sistema Iberoamericano de Información Judicial (SIIJ). 
En varias CCJ se destacó la celebración de la Cumbre Judicial Iberoamericana 2014, en Chile, siendo que la cooperación judicial es un eje fundamental de ese foro internacional. Dicha cumbre es, como es sabido, la mayor organización mundial a nivel judicial, la cual vertebra la cooperación y concertación entre los poderes judiciales de los veintitrés países de la comunidad iberoamericana de naciones.

En igual sentido, en las actas de las CCJ se han hecho constar numerosas propuestas, las cuales -entre otras, como en el caso de lo señalado en la reunión celebrada en Río Hondo ${ }^{13}$ - consistieron en las siguientes: promover una mayor difusión y participación de los agentes interesados en la cooperación judicial; promover la utilización de las plataformas de información e interacción judicial de la web, tales como IBERRED, IBERJUS, etc. y la de los poderes judiciales de ambos países; continuar revisando las estadísticas binacionales sobre Cooperación Jurídica; Sugerir la conveniencia de continuar organizando Seminarios Judiciales; propiciar la participación de magistrados de ambos países; evaluar por las autoridades correspondientes la factibilidad de las comunicaciones judiciales y considerar desarrollar un manual de buenas prácticas en materia de cooperación jurídica bilateral.

\section{Algunos datos estadísticos}

Cabe resaltar que en el trabajo desarrollado en las CCJ se destaca el análisis de las estadísticas bilaterales, de las cuales se desprende que existe un aumento sostenido en la actividad judicial entre ambos países.

En tal sentido, y por vía ejemplar, cabe hacer referencia a la estadística oficial ${ }^{14}$ sobre exhortos internacionales, registrados en 2011 y 2012. Allí, Argentina figura ocupando el $50 \%$ de las solicitudes al concluir 2011, y con el $44 \%$ hasta mediados de 2012, aclarándose que ambas sumas consideran el total de requerimientos judiciales recibidos en Chile desde el exterior.

Allí sesionó el Comité de Integración Atacalar, el 26 y 27 de agosto de 2013.

Información emanada de la Excma. Corte Suprema de Chile. 


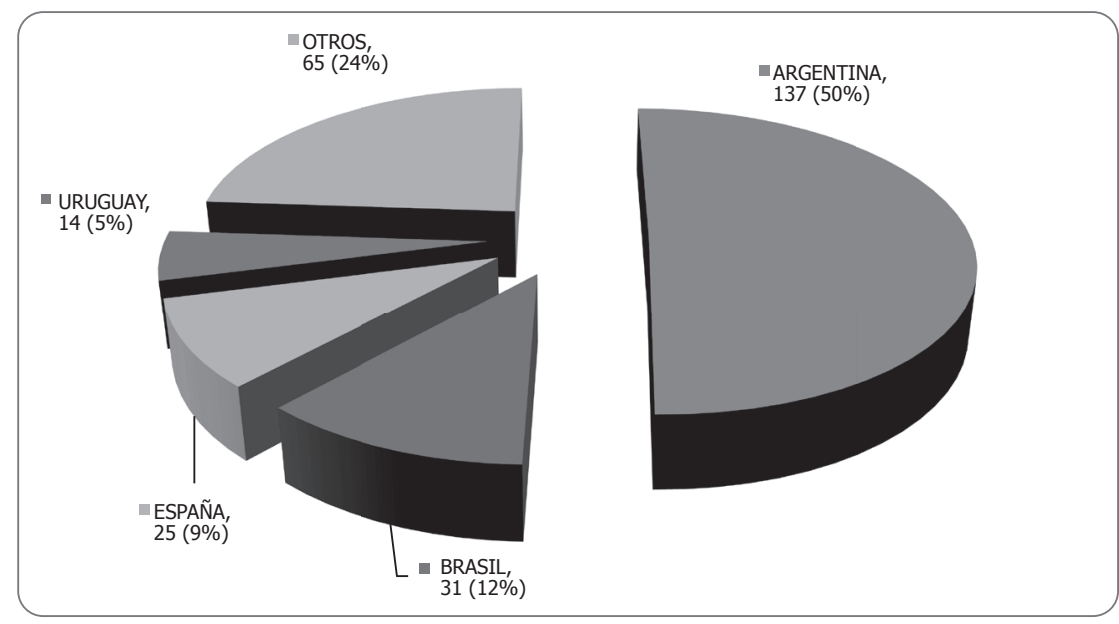

Gráfico 1. Exhortos 2011, del exterior a Chile

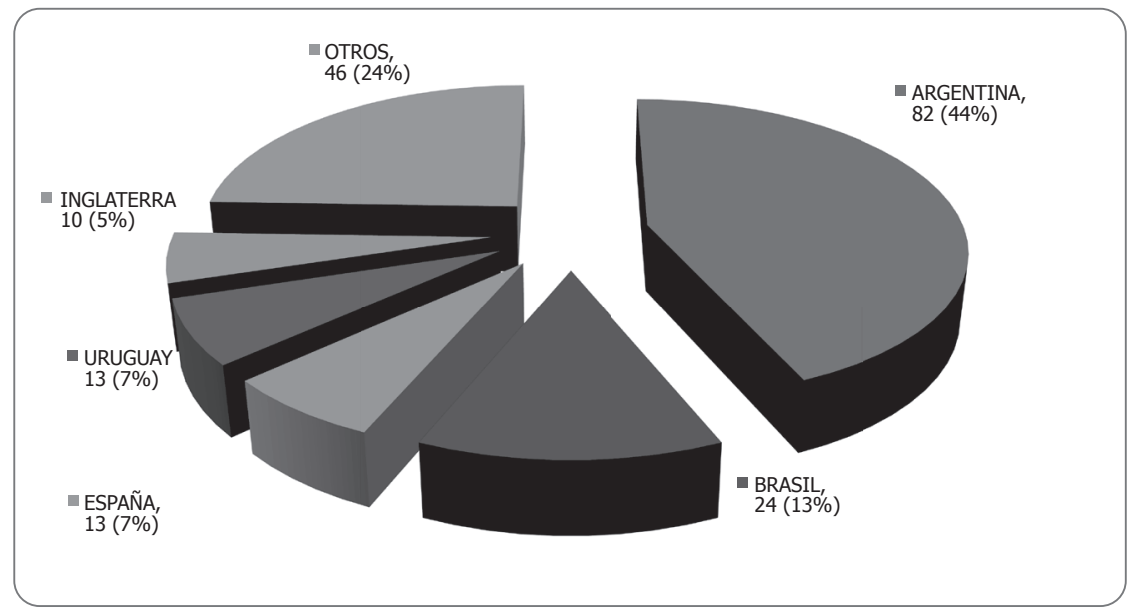

Gráfico 2. Exhortos a agosto de 2012, del exterior a Chile

En lo que dice relación con la naturaleza penal de las solicitudes cursadas entre ambos Estados $^{15}$, en el periodo 2004-2012 resalta el factor de los requerimientos pasivos (provenientes desde Argentina), los que ocupan el $47 \%$ del total, lo cual contrasta con el 24\% de requerimientos activos (generados desde Chile).

15 Fuente: Ministerio Público chileno. 
Lo anterior se puede apreciar en el siguiente gráfico:

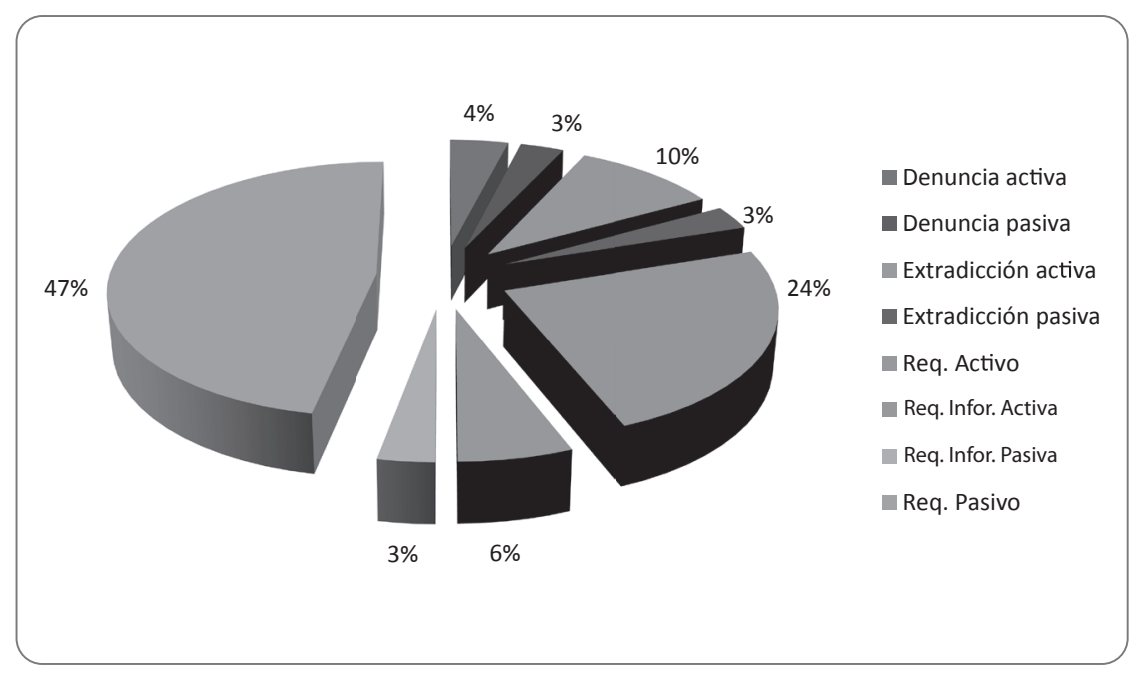

Gráfico 3. Requerimiento según tipo 2004-2012

\section{Conclusiones}

Como se ha podido observar, la cooperación judicial desde el enfoque subnacional, la cual respeta los lineamientos del Tratado de Maipú, no es sólo un tema emergente sino que, además, uno auspicioso.

Según se detalló, tanto por la temática abordada como por las conclusiones recogidas en las CCJ y seminarios, se hace evidente que estas nuevas y modestas instancias (que no poseen rol normativo, ni resolutivo) permiten generar jornadas de reflexión, discusión y elaboración de ideas útiles para seguir fortaleciendo los mecanismos de cooperación judicial ya existentes a nivel bilateral, sobre la base de un adecuado diagnóstico de la realidad tanto local como nacional.

Resulta pues un hecho que las diversas actividades descritas, junto con permitir avances en este tópico, generan nuevos vínculos institucionales y la profundización de confianzas mutuas, elementos que resultan claves al momento de cimentar adecuadamente 
cualquier nuevo esfuerzo de mayor integración. Ya lo decía el autor de estas iniciativas: "más y mejor integración es el desafío de nuestro tiempo, y sin duda esta debe ser la propuesta política de los próximos años" ${ }^{16}$.

En tales circunstancias, los seminarios y las CCJ pueden entenderse como elementos que suman en el proceso encabezado por las cancillerías, puesto que han enriquecido la discusión sobre cooperación judicial, al aportarles a ésta, la óptica de la realidad y especificidades de cada área geográfica.

Adicionalmente, estas instancias han permitido vincular a los órganos públicos y privados que poseen interés y/o competencias específicas en la cooperación, y entre los propios órganos jurisdiccionales de ambos países, generándose próximamente nuevos intercambios de experiencias, de profesionales y de información. En otras palabras, se abren nuevas puertas para que florezcan renovados conceptos e intereses comunes.

Es así que con estas actividades se ha generado un universo de unos doscientos funcionarios interesados en participar de la temática, promoviéndose el conocimiento personal entre los magistrados de ambos Estados. Este factor hará fluir más fácilmente ideas y propuestas de cambios, para perfeccionar la relación judicial, ya que le agrega un contendido personal a las relaciones institucionales ya descritas.

Cabe consignar, asimismo, el alto nivel de los representantes de cada una de las instituciones presentes en las diferentes CCJ y seminarios, lo que ha sido una clara muestra de la importancia que tales instituciones le han dado a estos encuentros, destacándose el gesto de la Corte Suprema chilena de enviar a sus más altos jueces a más de una decena de estas reuniones.

No podríamos dejar de afirmar, finalmente, que el Derecho en todas sus expresiones e incluido su semblante judicial, que de momento resulta básico en todo proceso de integración, indudablemente brindará todos los frutos esperados y permitirá consolidar el éxito de la política integracionista de ambos Estados sudamericanos.

16 Zaldívar Larraín, Adolfo. Integración Chile-Argentina; Desafio del Siglo XXI. El Ateneo, Buenos Aires, 2011 , p. 6. 
